\title{
On-line packing and covering a disk with disks
}

\author{
Janusz Januszewski
}

Received: 9 September 2009 / Published online: 1 May 2011

(C) The Author(s) 2011. This article is published with open access at Springerlink.com

\begin{abstract}
A circular disk $D$ of area $|D|$ can be on-line covered with any sequence of circular disks of total area not smaller than $6.488|D|$. Furthermore, any sequence of circular disks whose total area does not exceed $0.197|D|$ can be on-line packed into $D$.
\end{abstract}

Keywords On-line covering · On-line packing $\cdot$ Disk

Mathematics Subject Classification (2000) $\quad 52 \mathrm{C} 15 \cdot 05 \mathrm{~B} 40$

\section{Introduction}

Let $C, C_{1}, C_{2}, \ldots$ be planar convex bodies. We say that the sequence $\left(C_{i}\right)$ permits a covering of $C$ if there exist rigid motions $\sigma_{i}$ such that $C \subseteq \bigcup \sigma_{i} C_{i}$. We say that $\left(C_{i}\right)$ can be packed into $C$ if there exist rigid motions $\sigma_{i}$ such that $\bigcup \sigma_{i} C_{i} \subseteq C$ and that $\sigma_{i} C_{i}$ have mutually disjoint interiors. The on-line restriction means that at the beginning we are given only the first set $C_{1}$; then we learn each successive set $C_{i}$ only after the motion $\sigma_{i-1}$ has been provided. The placement of each set $\sigma_{i} C_{i}$ cannot be changed afterwards.

The area of $C$ is denoted by $|C|$.

Let $D$ be a circular disk. Denote by $c(D)$ the least number such that any (finite or infinite) sequence of circular disks with total area greater than $c(D)|D|$ permits an on-line covering of $D$. Furthermore, denote by $p(D)$ the greatest number such that

J. Januszewski $(\bowtie)$

Institute of Mathematics and Physics, University of Technology and Life Sciences,

ul. Kaliskiego 7, 85-796 Bydgoszcz, Poland

e-mail: januszew@utp.edu.pl 
any (finite or infinite) sequence of circular disks with total area smaller than $p(D)|D|$ can be on-line packed into $D$.

In Dumitrescu and Jiang (2010) it is proved that $c(D)<9.763$. In this paper we show that $c(D)<6.488$. On the other hand, $c(D) \geq 2.25$; three disks of diameters smaller than the side length of an equilateral triangle inscribed in $D$ do not permit a covering of $D$.

Any sequence of squares with total area not greater than 1/3 can be on-line packed into the unit square (see Han et al. 2008). Obviously, any disk $D$ of diameter $d$ can be inscribed into a square $S$ of side length $d$ as well as $D$ contains an inscribed square $R$ of side length $d / \sqrt{2}$. Since $|S| /|D|=4 / \pi$ and $|D| /|R|=\pi / 2$ it follows that $p(D) \geq \frac{1}{3} \cdot \frac{1}{2}=\frac{1}{6} \approx 0.167$. On the other hand, $p(D) \leq 0.5$; two disks of diameters greater than a half of the diameter of $D$ cannot be packed into $D$. The aim of this paper is to show that $p(D)>0.197$.

Other results concerning on-line packings and coverings are given in Kuperberg (1994), Lassak (1997) and Lassak and Zhang (1991).

By $[a, b] \times[c, d]$ we mean $\{(x, y) ; a \leq x \leq b, c \leq y \leq d\}$.

\section{The method of the first free subbrick}

In Sect. 3 we will use the method of the first free subbrick introduced in Januszewski and Lassak (1997). Let

$$
\lambda=\left[\frac{1}{17}\left(7-4 \cdot 2^{1 / 2}\right)\right]^{1 / 2} \approx 0.281 .
$$

By a brick of size $p$, where $p \in\{0,1,2, \ldots\}$, we mean a rectangle of side lengths $\lambda 2^{(3-p) / 2}, \lambda 2^{(2-p) / 2}$.

We can dissect any brick of size $p$ into two congruent bricks, called subbrick, of size $p+1$. Consequently, any brick of size $p$ is dissected into $2^{q}$ subbricks of size $p+q$. A brick of size 0 is also called a subbrick.

Let

$$
\begin{aligned}
& K_{1}=[-\lambda / 2, \lambda / 2] \times[\lambda, \lambda+\lambda \sqrt{2} / 2], \\
& K_{2}=[-\lambda / 2, \lambda / 2] \times[-\lambda-\lambda \sqrt{2} / 2,-\lambda], \\
& K_{3}=[-\lambda \sqrt{2}, \lambda \sqrt{2}] \times[-\lambda, \lambda] .
\end{aligned}
$$

Obviously, both $K_{1}$ and $K_{2}$ are bricks of size 3 and $K_{3}$ is a brick of size 0 .

Assume that $B_{1}, B_{2}, \ldots$ is a sequence of bricks. We will pack these bricks into $K_{1} \cup K_{2} \cup K_{3}$.

Let $q$ be an arbitrary non-negative integer. We enumerate all $2^{q}$ subbricks of $K_{1}$ of size $3+q$ by integers from 1 to $2^{q}$. In particular, $K_{1}$ is the first brick of size 3 . We enumerate all $2^{q}$ subbricks of $K_{2}$ of size $3+q$ by integers from 1 to $2^{q}$. Finally, we enumerate all $2^{q}$ subbricks of $K_{3}$ of size $q$ by integers from 1 to $2^{q}$. The only requirement is that the integers $2 m-1$ and $2 m$ are given to the subbricks of size $s+1$ of the subbrick of size $s$ whose number is $m$. 
By a free subbrick we mean a subbrick whose interior has an empty intersection with any brick packed before. For each $B_{i}$ from the sequence we find the smallest integer $j \in\{1,2,3\}$ such that there is a free subbrick of $K_{j}$ congruent to $B_{i}$. We pack $B_{i}$ into the congruent free subbrick of $K_{j}$ with the smallest possible number.

We stop the packing process if a subbrick $B_{z}$ from the sequence cannot be packed into $K_{1} \cup K_{2} \cup K_{3}$.

\section{On-line disks packing}

Theorem 1 Any sequence of circular disks whose total area does not exceed

$$
\frac{35-20 \sqrt{2}}{34}|D| \approx 0.1975|D|
$$

can be on-line packed into a circular disk $D$.

Proof Without loss of generality we can assume that $D$ is a disk with radius $1 / 2$ centered at the origin.

Let $D_{1}, D_{2}, \ldots$ be a sequence of disks and let

$$
\sum\left|D_{i}\right| \leq \frac{35-20 \sqrt{2}}{34}|D|=\frac{5}{2} \lambda^{2}|D| .
$$

Denote by $d_{i}$ the diameter of $D_{i}$.

It is easy to see that any $D_{i}$ is contained in a brick $B_{i}$ with the longest side shorter than $2 d_{i}$. Consequently, by $\left|D_{i}\right|=\frac{1}{4} \pi d_{i}^{2}$ and $\sqrt{2} d_{i} \cdot 2 d_{i}>\left|B_{i}\right|$ we have

$$
\left|D_{i}\right|>\frac{\pi \sqrt{2}}{16}\left|B_{i}\right|
$$

Since

$$
\left(\frac{1}{2} \lambda\right)^{2}+\left(\lambda+\frac{\sqrt{2}}{2} \lambda\right)^{2}=\frac{1}{4}
$$

and

$$
(\lambda \sqrt{2})^{2}+\lambda^{2}<\frac{1}{4}
$$

it follows that $D$ contains the bricks $K_{1}, K_{2}, K_{3}$.

We pack $B_{i} \supset D_{i}$ into $K_{1} \cup K_{2} \cup K_{3} \subset D$ by the method of the first free subbrick.

Contrary to the statement assume that it is impossible to pack $D_{1}, D_{2}, \ldots$ into $D$ by this method. Let $D_{z}$ be the disk which stops the packing process. We show that this leads to the false inequality 


$$
\sum_{i=1}^{z}\left|D_{i}\right|>\frac{5}{2} \lambda^{2}|D|
$$

Obviously, $\left|D_{i}\right|=\frac{1}{4} \pi d_{i}^{2} \leq \frac{5}{2} \lambda^{2}|D|=\frac{5}{2} \lambda^{2} \cdot \frac{1}{4} \pi$ and, consequently, $d_{i}<2 \lambda$, for each $i \in\{1,2, \ldots, z\}$. Hence at least one disk is packed into $K_{3}$. Consider two cases.

Case 1. Either $d_{z} \leq \lambda \sqrt{2} / 2$ or there is a disk of diameter smaller than or equal to $\lambda \sqrt{2} / 2$ packed into $K_{3}$. By the description of the packing method we deduce that at least one brick has been packed into $K_{1}$ and at least one brick has been packed into $K_{2}$ as well.

By the Proposition of Januszewski and Lassak (1997) we conclude that

$$
\sum_{i=1}^{z}\left|B_{i}\right|>\left|K_{1}\right|+\left|K_{2}\right|+\left|K_{3}\right|=5 \sqrt{2} \lambda^{2}
$$

Consequently,

$$
\sum_{i=1}^{z}\left|D_{i}\right|>\frac{\pi \sqrt{2}}{16} \sum_{i=1}^{z}\left|B_{i}\right|=\frac{\pi \sqrt{2}}{16} \cdot 5 \sqrt{2} \lambda^{2}=\frac{5}{8} \lambda^{2} \pi=\frac{5}{2} \lambda^{2}|D|
$$

Case 2. There is no disk of diameter smaller than or equal to $\lambda \sqrt{2} / 2$ packed into $K_{3}$ and $d_{z}>\lambda \sqrt{2} / 2$.

Subcase $2 A: d_{z}>\sqrt{2} \lambda$.

There is at least one disk (of diameter greater than $\lambda \sqrt{2} / 2$ ) packed into $K_{3}$. Consequently,

$$
\sum_{i=1}^{z}\left|D_{i}\right|>\pi\left(\frac{\sqrt{2} \lambda}{4}\right)^{2}+\left|D_{z}\right|>\pi\left(\frac{\sqrt{2} \lambda}{4}\right)^{2}+\pi\left(\frac{\sqrt{2} \lambda}{2}\right)^{2}=\frac{5}{2} \lambda^{2}|D| .
$$

Subcase $2 B: \lambda<d_{z} \leq \sqrt{2} \lambda$.

At most one subbrick of $K_{3}$ of size 2 is free. Consequently, at least three subbricks of $K_{3}$ of size 2 are not free and

$$
\sum_{i=1}^{z}\left|D_{i}\right| \geq \frac{\pi \sqrt{2}}{16} \cdot 3 \sqrt{2} \lambda^{2}+\left|D_{z}\right|>\frac{\pi \sqrt{2}}{16} \cdot 3 \sqrt{2} \lambda^{2}+\pi\left(\frac{\lambda}{2}\right)^{2}=\frac{5}{2} \lambda^{2}|D| .
$$

Subcase $2 C: \lambda \sqrt{2} / 2<d_{z} \leq \lambda$. There is no free subbrick of $K_{3}$. Consequently,

$$
\sum_{i=1}^{z}\left|D_{i}\right| \geq \frac{\pi \sqrt{2}}{16} \cdot 4 \sqrt{2} \lambda^{2}+\left|D_{z}\right|>\frac{\pi \sqrt{2}}{16} \cdot 4 \sqrt{2} \lambda^{2}+\pi\left(\frac{\sqrt{2} \lambda}{4}\right)^{2}=\frac{5}{2} \lambda^{2}|D| .
$$




\section{A method of covering with squares}

Let $S_{i}$ be a square of side length $s_{i}$ smaller than 1 , for $i=1,2, \ldots$. Let $R_{i} \subset S_{i}$ be a rectangle of width $w_{i}$ and of height $s_{i}$, where $w_{i} \leq s_{i}<2 w_{i}$ and where $w_{i} \in\left\{2^{-1}, 2^{-2}, 2^{-3}, \ldots\right\}$.

Denote by $P_{l}$ and $P_{r}$ the following sets:

$$
\begin{aligned}
& P_{l}=([-0.5,0] \times[-0.5,0.5]) \backslash([-0.5,-0.25] \times[-0.5,-0.25 \sqrt{3}]), \\
& P_{r}=([0,0.5] \times[-0.5,0.5]) \backslash([0.25,0.5] \times[-0.5,-0.25 \sqrt{3}]) .
\end{aligned}
$$

We describe an on-line method of covering of $P_{l} \cup P_{r}$ with $S_{1}, S_{2}, \ldots$. This method is a modification of the method presented in Januszewski (2009).

The squares $S_{i} \supset R_{i}$ will be divided into two types depending on the width of $R_{i}$. If $w_{i} \in\left\{2^{-1}, 2^{-3}, 2^{-5}, \ldots\right\}$, then $S_{i}$ is an $l$-square.

If $w_{i} \in\left\{2^{-2}, 2^{-4}, 2^{-6}, \ldots\right\}$, then $S_{i}$ is an $r$-square.

The description of the covering method is inductive.

Let $i \geq 1$. Assume that if $i>1$, then the motions $\sigma_{1}, \ldots, \sigma_{i-1}$ have been provided and that the sets $Q_{1}, \ldots, Q_{i-1}$ have been defined.

Let $q \in\{l, r\}$. Denote by $b_{i}^{q}$ the greatest number not greater than 0.5 such that each point of $P_{q}$ whose $y$-coordinate is smaller than $b_{i}^{q}$ belongs to $\bigcup_{j=1}^{i-1} Q_{j}$ (if $i=1$, then the union of $Q_{j}$ is taken as an empty set and $b_{1}^{q}=-0.5$ ). The set of points of $P_{q}$ with $y$-coordinate $b_{i}^{q}$ is called the $i$ th $q$-bottom. A point $\left(x_{1}, y_{1}\right)$ of the $i$ th $q$-bottom is an $i q$-surface point if $x_{1} \neq 0.25$ and $x_{1} \neq-0.25$ and if no point $\left(x_{1}, y_{1}+\delta\right)$ belongs to $\bigcup_{j=1}^{i-1} Q_{j}$, for $\delta>0$. By an il-place we mean a set containing an $i l$-surface point which has the form

$$
\left\{(x, y) ;-0.5+k w_{i} \leq x \leq-0.5+(k+1) w_{i}, b_{i}^{l} \leq y \leq b_{i}^{l}+s_{i}\right\}
$$

where $k \in\left\{0,1, \ldots,\left(2 w_{i}\right)^{-1}-1\right\}$. By an ir-place we mean a set containing an ir -surface point which has the form

$$
\left\{(x, y) ; k w_{i} \leq x \leq(k+1) w_{i}, b_{i}^{r} \leq y \leq b_{i}^{r}+s_{i}\right\}
$$

where $k \in\left\{0,1, \ldots,\left(2 w_{i}\right)^{-1}-1\right\}$.

$S_{i}$ is a $q$-square, where either $q=l$ or $q=r$. If $P_{q}$ is not covered yet, then we take $v=q$. Otherwise, we take $v=l$ provided $q=r$ and we take $v=r$ provided $q=l$. We say that $S_{i}$ is used for the covering of $P_{v}$.

(1) If $b_{i}^{v}<0.5-s_{i}$, then $S_{i}$ is a basic square. Furthermore, $R_{i}$ is a basic rectangle. We move $S_{i} \supset R_{i}$ so that $\sigma_{i} R_{i}$ is located in an $i v$-place.

The part of $S_{i}$ used for the covering is defined as

$$
Q_{i}=\left(P_{v} \cap \sigma_{i} R_{i}\right) \backslash \bigcup_{j=1}^{i-1} Q_{j}
$$


(if $i=1$, then the union of sets $Q_{j}$ is taken as an empty set). Obviously, if either $w_{i} \leq 0.25$ or $w_{i}=0.5$ and, at the same time, $b_{i}^{v} \geq-0.25 \sqrt{3}$, then $P_{v} \cap \sigma_{i} R_{i}=\sigma_{i} R_{i}$.

(2) If $b_{i}^{v} \geq 0.5-s_{i}$, then $S_{i}$ is a special square.

Take $k_{l}=-0.5$ provided the point $(-0.5,0.5)$ is not covered by any special square preceding $S_{i}$. Otherwise, denote by $k_{l}$ the greatest number such that any point of the segment $\left[-0.5, k_{l}\right] \times[0.5,0.5]$ is covered by a special square preceding $S_{i}$.

Take $k_{r}=0.5$ provided the point $(0.5,0.5)$ is not covered by any special square preceding $S_{i}$. Otherwise, denote by $k_{r}$ the smallest number such that any point of the segment $\left[0.5-k_{r}, 0.5\right] \times[0.5,0.5]$ is covered by a special square preceding $S_{i}$.

If $v=l$, then $\sigma_{i} S_{i}=\left[k_{l}, k_{l}+s_{i}\right] \times\left[0.5,0.5-s_{i}\right]$.

If $v=r$, then $\sigma_{i} S_{i}=\left[0.5-k_{r}-s_{i}, 0.5-k_{r}\right] \times\left[0.5,0.5-s_{i}\right]$.

Furthermore, we take $Q_{i}=\emptyset$.

\section{On-line covering with disks}

Let $q \in\{l, r\}$. Moreover, let $q^{*}=l$ if $q=r$ and $q^{*}=r$ if $q=l$. Assume that $S_{i}$ is a basic $q$-square used for the covering of $P_{q^{*}}$. By Lemma 1 of Januszewski (2009) we deduce that the area of the part of $S_{i}$ used for the covering exceeds $\frac{1}{3}\left|S_{i}\right|$, i.e.,

$$
\left|S_{i}\right|<3\left|Q_{i}\right|
$$

Lemma Assume that $S_{i}$ is a basic q-square used for the covering of $P_{q}$ and that $P_{q^{*}}$ is not covered by the squares preceding $S_{i}$. The area of the part of $S_{i}$ used for the covering exceeds $\frac{2}{5}\left|S_{i}\right|$, i.e., $\left|S_{i}\right|<\frac{5}{2}\left|Q_{i}\right|$.

Proof If $\sigma_{j} R_{j} \subset P_{q}$ for $j<i$, then $S_{j}$ is a $q$-square. Hence no basic rectangle of width $\frac{1}{2} w_{i}$ has a non-empty intersection with Int $\sigma_{i} R_{i}$. At most three basic rectangles of width $\frac{1}{4} w_{i}$ (and of height smaller than $\frac{1}{2} w_{i}$ ) have a non-empty intersection with Int $\sigma_{i} R_{i}$. The part of $\sigma_{i} R_{i}$ covered with basic rectangles preceding $R_{i}$ is of area smaller than

$3 \cdot \frac{1}{4} w_{i} \cdot \frac{1}{2} w_{i}+3 \cdot \frac{1}{16} w_{i} \cdot \frac{1}{8} w_{i}+\cdots=\frac{3 w_{i}^{2}}{8}\left(1+\frac{1}{16}+\frac{1}{64} \cdots\right)=\frac{3 w_{i}^{2}}{8} \cdot \frac{16}{15}=\frac{2}{5} w_{i}^{2}$.

It is easy to see that

$$
\left|Q_{i}\right|>\left|R_{i}\right|-\frac{2}{5} w_{i}^{2}=w_{i} s_{i}-\frac{2}{5} w_{i}^{2}
$$

Since

$$
\frac{2}{5} s_{i}^{2}-w_{i} s_{i}+\frac{2}{5} w_{i}^{2}<0
$$

for $w_{i} \leq s_{i}<2 w_{i}$, it follows that $\left|Q_{i}\right|>\frac{2}{5} s_{i}^{2}=\frac{2}{5}\left|S_{i}\right|$. 
Theorem 2 A circular disk D can be on-line covered with any sequence of circular disks whose total area is not smaller than

$$
(8.125+0.6875 \sqrt{3}-2 \sqrt{2})|D| \approx 6.487|D| .
$$

Proof Let $D$ be a circular disk and let $\left(D_{i}\right)$ be a sequence of circular disks with total area greater than or equal to

$$
(8.125+0.6875 \sqrt{3}-2 \sqrt{2})|D| .
$$

We lose no generality in assuming that $D$ is a disk with radius 0.5 centered at the origin.

It is easy to see that $D$ is contained in $P_{l} \cup P_{r}$. Furthermore, any $D_{i}$ contains an inscribed square $S_{i}$ of side length $s_{i}$ such that $\left|D_{i}\right| /\left|S_{i}\right|=\pi / 2$. Hence

$$
\sum\left|S_{i}\right|=\frac{2}{\pi} \sum\left|D_{i}\right| \geq 0.5(8.125+0.6875 \sqrt{3}-2 \sqrt{2}) \approx 3.24 .
$$

We can assume that the diameter $d_{i}$ of each $D_{i}$ is smaller than 1 ; otherwise $D$ can be covered by $D_{i}$. Consequently, we can assume that the side length of each $S_{i}$ is smaller than $0.5 \sqrt{2}$.

We cover $P_{l} \cup P_{r}$ by $S_{1}, S_{2}, \ldots$ by the method described in Sect. 4. Obviously, if $P_{l} \cup P_{r} \subseteq \bigcup \sigma_{i} S_{i}$, then $D \subset \cup \sigma_{i} D_{i}$.

We prove Theorem 2 indirectly. Assume that we apply the covering method and that $P_{l} \cup P_{r}$ is not contained in $\bigcup \sigma_{i} S_{i}$. We show that this leads to the false inequality

$$
\sum\left|S_{i}\right|<0.5(8.125+0.6875 \sqrt{3}-2 \sqrt{2})
$$

Consider three cases. Take $a=0.5-0.25 \sqrt{3}$.

Case 1. Neither $P_{l}$ nor $P_{r}$ is covered.

The parts of the basic squares used for the covering are pairwise disjoint and they are contained in $P_{l} \cup P_{r}$. By the Lemma we deduce that the total area of the basic squares is not greater than $2.5\left|P_{l}\right|+2.5\left|P_{r}\right|$. The total area of the special squares is smaller than $0.5^{2}+0.5^{2}$. Hence the total area of the squares is smaller than

$$
2.5(0.5-0.25 a)+2.5(0.5-0.25 a)+0.5=3-1.25 a<3
$$

which is a contradiction.

Case 2. $P_{l}$ is covered.

Let $S_{j}$ be the last special square used for the covering of $P_{l}$. The total area of the squares used for the covering of $P_{l}$ is smaller than

$$
2.5\left|P_{l}\right|+0.5^{2}+s_{j}^{2}
$$

Subcase $2 A$. The point $(0.5,0.5)$ belongs to $\sigma_{j} S_{j}$. Obviously, $s_{j}>0.5$. 
Since $P_{r}$ is not covered, it follows that $b_{n}^{r}<0.5-s_{j}$ for each positive integer $n$ such that $S_{n}$ has been used for the covering of $P_{r}$. The total area of parts of basic rectangles lying above the $n$th $r$-bottom (for any positive integer $n$ ) is smaller than

$$
\frac{1}{4} \cdot \frac{1}{2}+\frac{1}{8} \cdot \frac{1}{4}+\cdots=\frac{1}{8}\left(1+\frac{1}{4}+\cdots\right)=\frac{1}{8} \cdot \frac{4}{3}=\frac{1}{6} .
$$

Thus the total area of the squares used for the covering of $P_{r}$ is smaller than

$$
3\left[0.5\left(1-s_{j}\right)+\frac{1}{6}\right]+0.5^{2}
$$

Consequently, the total area of the squares in the sequence is smaller than

$$
2.5(0.5-0.25 a)+0.25+s_{j}^{2}+1.5-1.5 s_{j}+0.5+0.25
$$

It is easy to check that this upper bound is smaller than 3.21 provided $0.5<s_{j}<$ $0.5 \sqrt{2}$, which is a contradiction.

Subcase $2 B$. The point $(0.5,0.5)$ does not belong to $\sigma_{j} S_{j}$.

First assume that the special squares have pairwise disjoint interiors.

The total area of the bottom squares is not greater than

$$
2.5\left|P_{l}\right|+3\left|P_{r}\right|=5.5(0.5-0.25 a)
$$

The special squares have pairwise disjoint interiors and they have side lengths smaller than $0.5 \sqrt{2}$. Moreover, the sum of $s_{m}$, taken over all indexes $m$ such that $S_{m}$ is a special square, is smaller than 1. It is easy to check that the total area of the special squares is smaller than $(0.5 \sqrt{2})^{2}+(1-0.5 \sqrt{2})^{2}$. Hence the total area of the squares is smaller than

$$
5.5(0.5-0.25 a)+(0.5 \sqrt{2})^{2}+(1-0.5 \sqrt{2})^{2}=0.5(8.125+0.6875 \sqrt{3}-2 \sqrt{2}),
$$

which is a contradiction.

Assume that there is a special square $S_{m}$ used for the covering of $P_{r}$ such that Int $S_{j} \cap$ Int $S_{m} \neq \emptyset$. Obviously, $s_{j}<s_{m}<0.5$, otherwise $P_{l} \cup P_{r}$ is covered. Furthermore, since $P_{r}$ is not covered, it follows that $b_{n}^{r}<0.5-s_{j}$ for any integer $n$ such that $S_{n}$ has been used for the covering of $P_{r}$.

At this point we show that the total area of the squares used for the covering of $P_{r}$ is smaller than $1.75-s_{j}^{2}$. Denote by $k$ the integer such that $2^{-k} \leq s_{j}<2^{-k+1}$. Let

$$
U_{1}=\left[0,2^{-k}\right] \times\left[0.5-s_{j}, s_{j}\right], \quad U_{2}=\left[2^{-k}, 2 \cdot 2^{-k}\right] \times\left[0.5-s_{j}, s_{j}\right] .
$$

There is $t \in\{1,2\}$ such that each basic rectangle of width not smaller than $2^{-k}$ has an empty intersection with Int $U_{t}$, otherwise $P_{r}$ is covered. Consequently, the area 
of the part of $U_{t}$ covered with basic rectangles does not exceed

$$
2^{-k-1} \cdot 2^{-k}+2^{-k-2} \cdot 2^{-k-1}+\cdots=\frac{2}{3} \cdot 2^{-2 k}
$$

This implies that the total area of the basic rectangles used for the covering of $P_{r}$ does not exceed

$$
\frac{1}{2}-\left|U_{t}\right|+\frac{2}{3} \cdot 2^{-2 k}
$$

Hence the sum of areas of all basic squares used for the covering of $P_{r}$ is not greater than

$$
3\left(\frac{1}{2}-\left|U_{t}\right|+\frac{2}{3} \cdot 2^{-2 k}\right)=\frac{3}{2}+2 \cdot 2^{-2 k}-3 \cdot 2^{-k} s_{j}
$$

Since $2^{-k} \leq s_{j}<2^{-k+1}$, it follows that this upper bound is not greater than $1.5-s_{j}^{2}$. Obviously, the total area of the special squares used for the covering of $P_{r}$ is smaller than $0.5^{2}$. Hence, the total area of the squares used for the covering of $P_{r}$ is smaller than $1.75-s_{j}^{2}$.

Consequently,

$$
\sum\left|S_{i}\right|<2.5(0.5-0.25 a)+0.5^{2}+s_{j}^{2}+1.75-s_{j}^{2}<3.21,
$$

which is a contradiction.

Case 3: $P_{r}$ is covered.

We argue in a way similar to the argument of Case 2.

The method described in Sect. 4 can be used also for the covering of

$$
I=[-0.5,0.5] \times[-0.5,0.5]
$$

with squares $S_{1}, S_{2}, \ldots$ In that case, if $I$ is not covered, then the total area of the squares in the sequence is smaller than $2.5 \cdot 0.5+3 \cdot 0.5+1=3.75$.

Corollary A unit square can be on-line covered with any sequence of squares whose total area is not smaller than 3.75 .

Open Access This article is distributed under the terms of the Creative Commons Attribution Noncommercial License which permits any noncommercial use, distribution, and reproduction in any medium, provided the original author(s) and source are credited.

\section{References}

Dumitrescu, A., Jiang, M.: Covering a disk by disks. Beitr. Algebra Geom. 51(1), 91-109 (2010) 
Han, X., Iwama, K., Zhang, G.: Online removable square packing. Theory Comput. Syst. 43(1), 38-55 (2008)

Januszewski, J.: On-line covering the unit square with squares. Bull. Pol. Acad. Sci. Math. 57(1), 57-62 (2009)

Januszewski, J., Lassak, M.: On-line packing sequences of cubes in the unit cube. Geom. Dedic. 67, 285-293 (1997)

Kuperberg, W.: On-line covering a cube by a sequence of cubes. Discret. Comput. Geom. 12, 83-90 (1994)

Lassak, M.: A survey of algorithms for on-line packing and covering by sequences of convex bodies, Bolyai Soc. Math. Stud. 6, János Bolyai Math. Soc., Budapest, pp. 129-157 (1997)

Lassak, M., Zhang, J.: An on-line potato-sack theorem. Discret. Comput. Geom. 6, 1-7 (1991) 